\title{
NTUs: An Intelligent Tutorial System Fosters Number Concepts Through Computational Scaffolding
}

\author{
Chih-Wei Hue Chien-Huei Kao Ming Lo Chien-Chih Chiang \\ Department of Psychology, National Taiwan University \\ Liong-Yu Tu and Wen-Lian Hsu \\ Institute of Computer Science, Academia Sinica
}

\begin{abstract}
The present article describes how scaffolding is implemented in an intelligent tutorial system call NTUs (Number Transcoding tUtorial System), and what are the results of the system tested empirically on a group of grade students in fostering their number concepts. To use NTUs. the system first analyzes a user's errors on a number transcoding task, and the results of analysis are used to infer the user's zone of proximal development (ZPD). A scaffolding process which was designed with inspiration from how people learn Chinese calligraphy will be provided next in the user's ZPD. Empirical test indicates that NTUs can not only foster students ' number concepts but also attract them to use it.
\end{abstract}

\section{1: Introduction}

A scaffold is a temporary framework designed to help workers to construct a building by providing supports to the building before it is strong enough to stand by itself. Wood, Bruner and Ross [1] used this kind of "scaffolding" process as a metaphor to illustrate how mothers assisted their young children to perform novel and complex tasks. According to Wood et al., in the scaffolding process, a mother will provide helps to her child on those elements of tasks, which are beyond the child 's capacity, and will allow the child to concentrate upon the elements which are within his/her range of competence.

The purpose of the present research was to test the effectiveness of an intelligent tutorial system (ITS) called NTUs (Number Transcoding tUtorial System) which was designed to foster grade students' number concepts. In particular, the system converts a number transcoding task into a game like situation, and adopts a computational scaffolding strategy to allow a user to develop number concepts gradually. In the following sections, the design of the system and the results the system tested in an experiment were discussed.

\section{2: The Design of NTUs}

To show clearly how NTUs is designed, the number transcoding task and the domain knowledge the task tested was discussed first, followed by how scaffolding processes were implemented in the system.

\section{1: The Number Transcoding Task}

Some researchers argued that the Chinese number system has a "regular" correspondence with the Arabic numeral system and that such correspondence facilitates 
Chinese children to learn number concepts and arithmetic [2]. For example, " 21 " is expressed as “er(2)-shi(10)-yi(1)" in Chinese. That is, Chinese number system is regular because usually each digit of a multiple-digit number is associated with two syllables, one represents the digit and the other represents the place value of the digit in the number. However, Hue [3] pointed out that although the Chinese number system is regular in general, there are two types of irregular number words in Chinese. One involves numbers containing consecutive "0"s. For example, "2002" is expressed as “er(2)-chien(1000)-lin(0)-er(2)". The two consecutive " 0 "s are expressed only once in the number word. In the second type, the place value of the rightmost non-zero digit of a multiple-digit number can be omitted in number word. Thus, "2200" can be expressed either as “er(2)-chien(1000)-er(2)-bai(100)" or as “er(2)-chien(1000)-er(2)" where the place value associated with 200 is omitted.

In an experiment, Hue [3] asked a group of grade students (from $2^{\text {nd }}$ to $6^{\text {th }}$ grade) to transcode Chinese number words into Arabic numbers. He found that (1) the students made more errors when they were transcoding irregular number words than regular ones, (2) the errors that the students made could be categorized into four broad categories similar to that found in previous literature [4], and in two of these categories, errors could be further divided into two types, each reflecting a special problem solving strategy used by students. More imp ortantly, Hue argued that each error category indicated that certain number concepts were not activated and used by students while transcoding the words. For example, there is a type of error called literal translation, where a number word is transcoded in to Arabic number character by character, e.g., “er(2)-chien(1000)-er(2) -bai(100)" (2200) is transcoded as 2000200. If a student committed such error it is likely that the student has problem with the following knowledge: the place value of a digit in Arabic number (the first " 2 " of 2000200 equals 2000000 , not 2000), carrying (10 “100”s equal 1000), and Chinese number word grammar (“er(2)-chien(1000) -er(2)-bai(100)" equals “er(2)-chien(1000)” plus “er(2)-bai(100)”).

\section{2: Computational Scaffolding}

To implement scaffolding in teaching, researchers usually adopt a social constructivist perspective and argue that successive scaffolding requires teachers to determining a student's "zone of proximal development" (ZPD), and scaffolding should be in the ZPD [5]. Although the concept of ZPD is easily understood conceptually, its definition is vague and usually post hoc in nature. For example, it was typically defined following Vygotsky's [6] original idea as "the distance between the child's actual developmental level as determined by independent problem solving and the higher level of potential development as determined through problem solving under adult guidance and in collaboration with more capable peers (pp. 67-68)" [7]. According to the definitions, it is difficult, if not impossible, to determine a child's ZPD before the child has been taught and tested. This creates a paradox for a teacher because in order to set up scaffolding process the teacher needs to know the students' ZPDs, however ZPD could only be determined after teaching.

The paradox of locating ZPD creates much difficulty when researchers try to design intelligent tutoring system to perform scaffolding, because a concept has to be "computational" to be programmed in computer and ZPD is certainly not "computational" if it is defined strictly according to Vygotsky's original idea. Although there are a few computerized learning environments providing 
"computational" scaffolding, the way ZPD was defined in these systems was just "spiritually" following Vygotsky's original idea. For example, in MEMOLAB [8], a learner goes through a sequence of microworlds, or levels, to gain knowledge of experimental psychology. Each level consists of a different set of psychological experiments, and a learner needs to learn a different set of commands in order to create or modify the experiments in a different level. Thus, ZPD is operationally defined as the new commands needed to move from level $\mathrm{L}$ to $\mathrm{L}+1$. According to Vygotsky's idea, one person's ZPD is likely to be different from another person, however, for all the learners, the commands used in the levels are the same and were assigned by the researchers.

The way NTUs handled the ZPD paradox was similar as MEMOLAB, only that NTUs was inspired by how human tutors interact with their tutees. Lepper, Drake and O'Donnell-Johnson [9] found that good tutors constantly alert to tutees' systematic errors, and that the tutors are continuously diagnosing, monitoring and fixing the misconceptions underlying tutees' errors. Thus, NTUs will infer a user's knowledge based on a pre-designed structure of number concepts and the errors the user made on a test. That is, the ZPD of a user is defined in NTUs as the deficient number concepts indicated in the errors the user made on the test. To access a user's ZPD and perform scaffolding, NTUs consists of four subsystems .

1. Knowledge representation system. The number concepts require to transcode correctly Chinese number words into Arabic numbers are represented in the system using the InfoMap algorithm designed by Hsu and his colleagues [10]. Because a number word can be categorized according to its regularity and the quantity it represents, the typology of Chinese number words is also represented in the system.

2. Problem posing system. The system writes outs problems for users to solve based on the knowledge represented in InfoMap. In NTUs, two problems are posed for each word type, and types of problem a user will receive depending on the grade $\mathrm{s} / \mathrm{he}$ is in. For a second grader, because the thousand concept has not been taught in school yet, s/he will receive only regular and the place-value missing problems. For a user who is in third grade or above, $\mathrm{s} / \mathrm{he}$ will receive both types of irregular problems, only that the numbers the word problems represented are within the range from 1000 to 9999 for a third grader, and for a fourth or fifth grader, the numbers are within the range between 10000 and 99999. The users interact with NTUs through two cartoon characters, a prince and his mentor, who are taking an adventurous journey to save a princess. In the journey, the prince has to compete with evil force in a number of computer games in order to arrive at the princess' castle. The problems that the problem posing system posed are embedded in the computer games.

3. Error diagnosis system. According to our previous studies, grade students are likely to make four types of systematic errors in a number transcoding task. The errors and the deficient number concepts indicated by the errors were represented in the system using InfoMap. The test results will be feed into the present system, and the errors along with their types and deficient number concepts with be computed. The results computed by the present system will feed to the teaching system so that scaffolding will proceed.

4. Teaching and scaffolding system. The design of the scaffolding process used in NTUs was inspired by how people learn to write Chinese calligraphy. A calligraphy master guides a new pupil to develop his/her writing style through four learning stages. In the "leading" stage, the master will ask the pupil to 
hold a brush pen, and he will hold the pupil's hand while writing Chinese character's "basic" strokes. The pupil will learn how to write the strokes by feeling how the master uses the brush. In "pattern tracing" stage, the master will write a few characters as patterns, and the pupil will trace the characters to learn how strokes are put together to form characters. In the "coping" stage, the pupil will imitate the writings of famous calligraphy maters, and learn further how strokes of a character should be arranged in the consideration of the figures of the characters surround ed it. Finally, in the "stylization" stage, the pupil will learn different writing styles and develop his/her own style.

In NTUs, when a user is diagnosed needed to learn certain number concept, the system will follow a procedure similar to how people learn to write Chinese calligraphy to build (and to fade away) scaffolds to allow the user to learn the concept. In the first stage, the computer "leads" the problem solving process and the user watches how each step the computer takes to solve a problem. In the second stage, in each step of the process to solve a problem, the computer will finish only part of it. The user needs to finish the rest. In the third stage, the computer will not solve the problem for the user, but only list the steps needed to solve it. The user needs to solve the problem all by himself/herself. Finally, the computer will generate problems and embedded them in different context for the user to solve.

In each scaffolding stage, the computer will check the user's answers. The teaching will not move to an advanced stage unless the user can solve correctly all the problems of the stage he is working on. For people who make different errors, the computer will assume that their knowledge of the number concepts is different, and they will receive different scaffolding materials although the scaffolding processes are the same.

\section{3: The Effectiveness of NTUs}

The NTUs was test on a group of $3^{\mathrm{d}}$ graders. The research consisted of two separate sessions. The 130 students were divided into two groups, and in the first session, both the control and the experimental group received diagnosis test using NTUs, however only the experimental group received scaffolding teaching. After 4 weeks of the first session, all the subjects received a paper and pencil test using another set of problems generated by the problem posing system and a questionnaire to indicate their attitudes toward the system. The results of the test and questionnaire were listed in Table 1 and 2, which indicated that NTUs is not only an effective way to foster students' number concepts but also an attractive educationware.

\begin{tabular}{|l|r|r|}
\hline & Experimental group & \multicolumn{1}{c|}{ Control group } \\
\hline Pre-test & $19.01 \%$ & $15.76 \%$ \\
\hline Post-test & $13.02 \%$ & $15.23 \%$ \\
\hline
\end{tabular}

Table 1: Pre- and post-scaffolding test results (percent of errors) of two groups of students on number transcoding tasks.

\begin{tabular}{|l|r|r|r|}
\hline Dimension & Negative & Neutral & \multicolumn{1}{c|}{ Positive } \\
\hline Cognition & $27.03 \%$ & $12.21 \%$ & $60.65 \%$ \\
\hline Affect & $8.43 \%$ & $35.17 \%$ & $46.51 \%$ \\
\hline Behavior & $8.14 \%$ & $0.0 \%$ & $91.87 \%$ \\
\hline
\end{tabular}

Table 2: The students' attitude toward NTUs, indicated in a 9 -item questionnaire. The items were grouped into the three dimensions of attitude.

\section{Conclusion}

The present research illustrated how the concepts of 
scaffolding are implemented in an ITS to foster users' number concepts. In particular, the design of NTUs is based on educational research of mathematical and Chinese calligraphy learning. Because the research is a part of a big project which involves designing educationware for school teachers, the results (students' performance on the test and their knowledge diagnoses) of the present system were presented to two experienced teachers for comments. They pointed out that NTUs not only could be used to infer a student's knowledge, but also that the diagnoses generated by the system provided much information concerning a student's number concepts which they heard the first time. They urged us to release the software, and are ready to incorporate the system into their normal teaching routine.

\section{References}

1. Wood, D.J., Bruner, J.S., \& Ross, G. (1976). The role of tutoring in problem solving. Journal of Child Psychology and Psychiatry, 17, 89-100.

2. Fuson, K.C., \& Kwon, Y. (1991). Chinese-based regular and Erropean irregular systems on number words: The disadvantages for English-speaking children. In K. Durkin \& B. Shire (eds.), Language and mathematical education (pp. 211-226). Milton Keynes, Great Britain: Open University Press.

3. Hue, C.W. (1997). Number transcoding: Effects of language on children's understanding of number concepts. Paper presented at the $13^{\text {th }}$ Science Education Conference of the Republic of China, Taipei, Taiwan.

4. Power, R. J. D. \& Dal Martello, M. F. (1990). The dictation of Italian numerals. Language and Cognitive Processes, 5, 237-254.

5. Roehler, L.R., \& Cantlon, D.J. (1997). Scaffolding: A powerful tool in social constructivist classrooms. In K. Hogan \& M. Pressley (eds.), Scaffolding student learning (pp. 6-42). Cambridge, MA: Brookline Books.

6. Vygotsky, L.S. (1978). Mind in society. Cambridge, MA: Harvard University Press.

7. Wertsch, J. (1985). Culture, communication, and cognition. Cambridge, England: Cambridge University Press.

8. Mendelsohn, P. (1996). Mapping models of cognitive development to design principles of learning environments. In S. Vosniadou, E. De Corte, R. Glaser, \& H. Mandl (eds.), International perspectives on the design of technology-supported learning environments (pp. 323-344). Mahwah, NJ: Erlbaum.

9. Lepper, M.R., Drake, M.F., \& ODonnell-Johnson, T. (1997). Scaffolding techniques of expert human tutors. In K. Hogan \& M. Pressley (eds.), Scaffolding student learning (pp. 108-144). Cambridge, MA: Brookline Books.

10. Hsu, W.L., Wu, S.H., \& Chen, Y.S. (2001). Event identification based on the information map INFOMAP. Paper presented at the IEEE International Workshop on Natural Language Processing and Knowledge Engineering in conjunction with the IEEE International Conference on Systems, Man and Cybernetics Conference, Tucson, Arizona, USA. 